\title{
Diagnóstico transoperatorio de situs inversus totalis en un paciente con apendicitis aguda complicada
}

\author{
Transoperative diagnosis of situs inversus totalis in a \\ patient with complicated acute appendicitis
}

\author{
Iris Natalia Cruz Zamudio, * Guillermo Patricio Campos Márquez, ${ }^{*}$ \\ Jorge Eduardo Espino Galván, ${ }^{\ddagger}$ Mario Vásquez Hernández ${ }^{\S}$
}

\section{Resumen}

Se presenta el caso clínico de un paciente masculino de 13 años con un cuadro clínico de apendicitis complicada y un diagnóstico transoperatorio de situs inversus totalis. Este caso clínico resalta la importancia de las variaciones anatómicas en una cirugía así y cómo afecta el diagnóstico oportuno de patologías quirúrgicas.

Palabras clave: Situs inversus totalis, apendicitis complicada, apendicectomía.

\section{INTRODUCCIÓN}

El dolor abdominal es uno de los principales motivos de consulta en urgencias, de ahí que se deban plantear múltiples diagnósticos diferenciales para llegar a uno definitivo. ${ }^{1}$ Debido a esto, la clínica es de vital importancia para llegar a un diagnóstico definitivo. La semiología puede ser confusa en pacientes con situs inversus totalis (SIT), una variante anatómica de baja incidencia. ${ }^{1}$ El propósito de este artículo es reportar un caso de apendicitis aguda complicada en un paciente con situs inversus totalis en el que la presentación

\footnotetext{
* Residente de segundo año de Cirugía General.

* Residente de tercer año de Cirugía General.

$\S$ Jefe de Servicio de Cirugía General.
}

Hospital General "Alfredo Pumarejo Lafaurie" de la Universidad Autónoma de Tamaulipas, Matamoros, Tamaulipas.

\section{Correspondencia:}

Iris Natalia Cruz Zamudio

correo electrónico: Nat_manzana@hotmail.es

Aceptado: 12-04-2019.

www.medigraphic.com/actamedica

\section{Abstract}

A case of a 13-year-old male patient with a clinical picture of complicated appendicitis with transoperative diagnosis of situs inversus is presented. This clinical case highlights the importance of anatomical variations in surgery as well as how it affects the timely diagnosis of surgical pathologies.

Keywords: Situs inversus totalis, complicated appendicitis, appendectomy.

clínica del caso conllevó a un diagnóstico transoperatorio de esta variable anatómica.

\section{CASO CLÍNICO}

Se trata de un paciente masculino de 13 años, que acudió al Servicio de Urgencias por presentar un cuadro de dolor abdominal de tres días y de evolución tipo cólico, el cual fue localizado en la fosa iliaca izquierda, y se irradia a la fosa iliaca derecha. Se evaluó el paciente con una escala EVA 10/10; su padecimiento está acompañado de náusea, vómito y anorexia, aunque fue atenuado con paracetamol.

En la exploración física encontramos al paciente en malas condiciones generales; sus signos vitales tenían marcada taquicardia, abdomen con peristalsis ausente en la palpación, "abdomen en tabla" y dolor en la palpación superficial y profunda de la fosa iliaca derecha; signo de Rovsing presente y Blumberg presente, además de percusión timpánica generalizada.

Dentro de sus exámenes de laboratorio encontramos a un paciente sin leucocitosis, sin embargo, con neutrofilia marcada (leucocitos 10.0 WBC 79.3), y el examen general de orina se presentó sin alteraciones. Integramos un síndrome de abdomen agudo, con diagnóstico probable de apendicitis aguda complicada, por lo que se decidió realizar 
una laparotomía exploradora en línea media infraumbilical, y se obtuvo como hallazgo transoperatorio trasposición de vísceras abdominales y apendicitis complicada con absceso pélvico (Figuras 1 y 2).

Se realizó la apendicectomía con técnica de Halsted y drenaje de absceso y no hubo incidentes. Durante el postoperatorio inmediato se solicitó una radiografía de tórax (Figura 3), la cual mostró dextrocardia, y el ultrasonido abdominal reportó transposición de las vísceras abdominales, confirmando el diagnóstico de situs inversus totalis. Al tercer día postoperatorio, el paciente presentó mejoría clínica y, al no presentar datos de complicaciones postoperatorias, se decide su egreso.

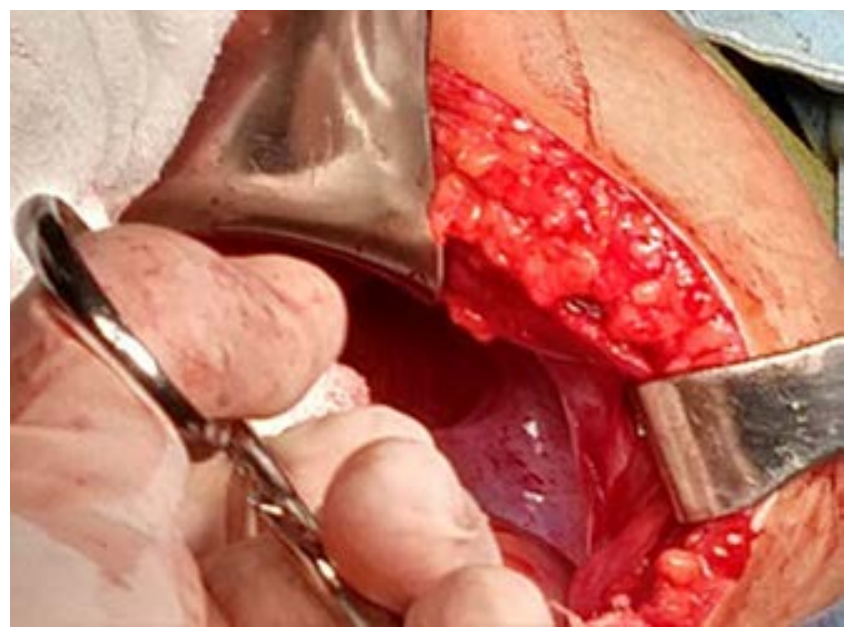

Figura 1: Hallazgos transoperatorios que muestran el hígado de localización izquierda.

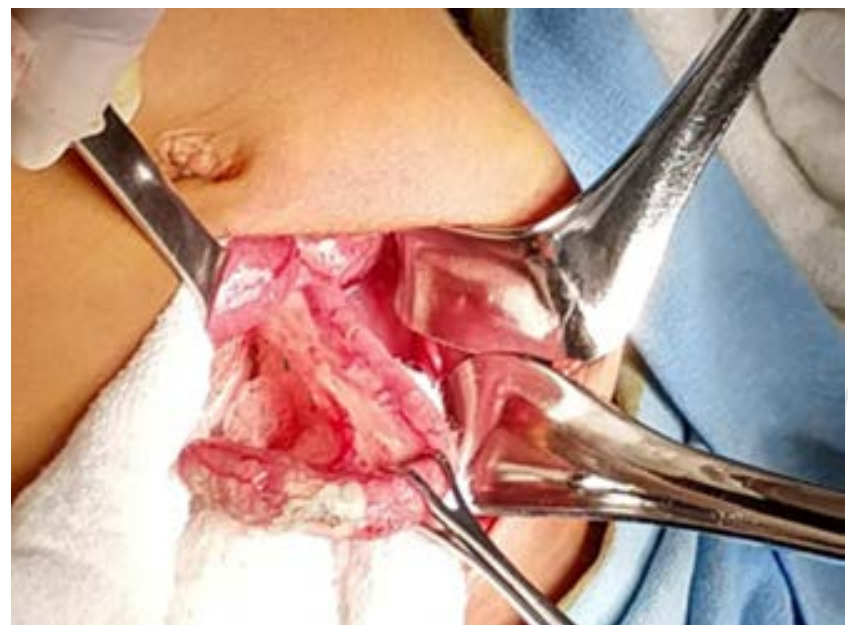

Figura 2: Hallazgos transoperatorios que muestran el apéndice cecal del lado izquierdo.

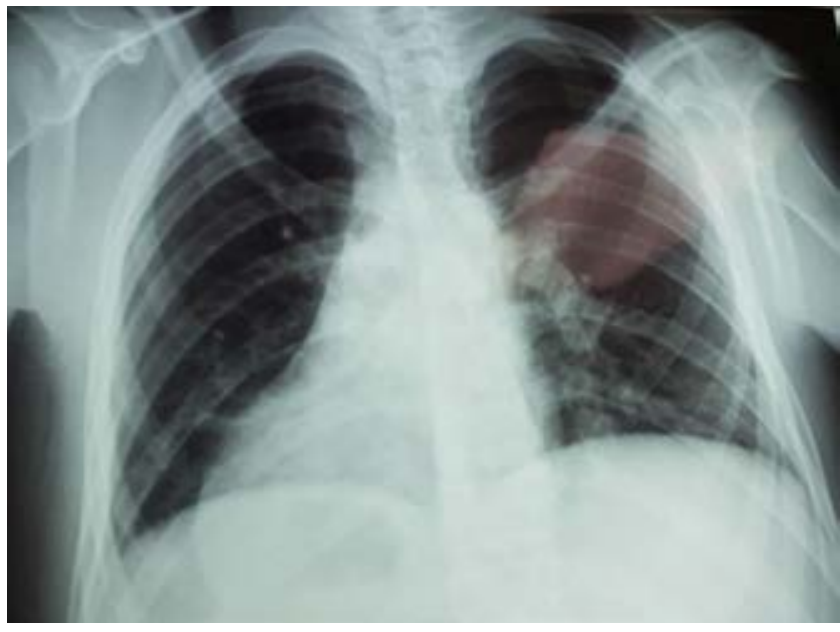

Figura 3: Radiografía de tórax que muestra dextrocardia.

\section{DISCUSIÓN}

La apendicitis aguda es la primera causa de abdomen agudo quirúrgico en la actualidad, constituyendo $40 \%$ de las causas de urgencias no traumáticas en los hospitales. ${ }^{1}$ La asociación de apendicitis aguda en un paciente con situs inversus es un gran reto diagnóstico y terapéutico. ${ }^{2}$ Descrito por primera vez en el año $1600,{ }^{2}$ el situs inversus totalis es una condición autosómica recesiva que involucra una transposición de los órganos abdominales y torácicos en espejo, y tiene una incidencia de 1:10,000 a 1:20,000. ${ }^{3}$

Normalmente la rotación embriológica del intestino sucede en un sentido antihorario, sin embargo, en el situs inversus ocurre en la dirección contraria. ${ }^{4}$ El origen de esta alteración es aún oscuro, aunque en la actualidad no se conoce su etiología específica; ${ }^{5}$ sin embargo, se propone que ésta es secundaria a una alteración genética de tipo autosómica recesiva en el brazo largo del cromosoma 14 y que afecta la cascada genética responsable de la diferenciación izquierda derecha. ${ }^{1}$ De igual manera se ha relacionado con una alteración en la función ciliar, ya que estos organelos están implicados en las migraciones celulares embrionarias y en la determinación de la lateralidad, iniciando así la simetría derecha-izquierda embrionaria. ${ }^{1}$

Por su lado, la apendicitis aguda es una de las causas más comunes de dolor abdominal y es un motivo frecuente de cirugía de urgencia. La coexistencia de estas dos patologías conlleva que el diagnóstico quirúrgico en estos pacientes pueda ser insidioso y secundario a la imagen en espejo de los órganos abdominales. ${ }^{3}$ La peritonitis por perforación de víscera hueca es extremadamente rara en el situs inversus; sólo se ha reportado un caso de perforación de vesícula 
biliar, un caso de perforación apendicular y dos casos de perforación de víscera hueca. ${ }^{3}$

El diagnóstico de situs inversus pasa inadvertido en aproximadamente $45 \%$ de los casos, y así se llega a su diagnóstico incidentalmente durante el transoperatorio o como hallazgo imagenológico. ${ }^{5}$ El ultrasonido abdominal, radiografía y tomografía de abdomen, confirman el diagnóstico. ${ }^{6} \mathrm{Al}$ igual que en nuestro caso clínico, el diagnóstico y los procedimientos quirúrgicos para las patologías abdominales en pacientes con situs inversus totalis son técnicamente más complicadas y conllevan un reto único debido a la transposición izquierdaderecha de las vísceras abdominales. ${ }^{7}$

\section{REFERENCIAS}

1. Rosiles-Domínguez C, Carrillo-Sánchez D, Lever-Rosas CD, SilvaOrtiz J et.al. Apendicetomía en paciente con situs inversus. Reporte de un caso, Rev. Sanid. Milit. Mex. 2013; 67 (3): 124-127.
2. Salama IA, Abdullah MH, Houseni M. Laparoscopic cholecystectomy in situs inversus totalis: Feasibility and review of literature. Int J Surg Case Rep. 2013; 4(8): 711-715. doi:10.1016/j.ijscr.2013.02.030

3. Ahmed Z, Khan SA, Chhabra $S$ et al. Our experience with surgery in situs inversus: Open peptic perforation repair and laparoscopic cholecystectomy in 1 patient and 3 patients respectively. Int J Surg Case Rep. 2016; 29: 34-38. doi:10.1016/j.ijscr.2016.10.035

4. Bostanci ME, Atabey M, Bozkurt B, Ozel I, Karadayi K. Co-existence of cecal volvulus with situs inversus totalis: A case report. Turk J Emerg Med. 2016; 16 (3): 134-135. Published 2016 Sep 29. doi:10.1016/j. tjem.2015.01.004

5. Melchor-González JM, Pérez-García R, Torres-Vista M, RodríguezBrambila VR. Situs Inversus. Reporte de dos casos. Cir Cir. 2000; 68: 72-75.

6. Sheng-ning Z, Jiang-Hua R et al. Laparoscopic Cholecystectomy in Situs Inversus Totalis: a Case Report. Chin Med Sci J 2013; 28 (4): 245-247.

7. Maurya AP1, Kumar S, Gupta V, Chandra A. Management of Complicated Choledochal Cyst in an Adult with Situs Inversus Totalis: Diagnostic Difficulties and Technical Notes. Indian J Surg. 2017; 79 (5): 464-467. doi: 10.1007/s12262-017-1613-3. Epub 2017 Mar 9. 